Case Report

\title{
Prosthetic Joint Infection due to Mycobacterium avium-intracellulare in a Patient with Rheumatoid Arthritis: A Case Report and Review of the Literature
}

\author{
Nicholas E. Ingraham, ${ }^{1}$ Brenton Schneider, ${ }^{2}$ and Jonathan D. Alpern ${ }^{3}$ \\ ${ }^{1}$ Department of Internal Medicine, University of Minnesota, Minneapolis, MN, USA \\ ${ }^{2}$ Department of Internal Medicine, Hennepin County Medical Center, Minneapolis, MN, USA \\ ${ }^{3}$ Department of Infectious Disease, University of Minnesota, Minneapolis, MN, USA
}

Correspondence should be addressed to Nicholas E. Ingraham; nicholas.ingraham@gmail.com

Received 26 September 2016; Accepted 24 January 2017; Published 9 February 2017

Academic Editor: Tomoyuki Shibata

Copyright (C) 2017 Nicholas E. Ingraham et al. This is an open access article distributed under the Creative Commons Attribution License, which permits unrestricted use, distribution, and reproduction in any medium, provided the original work is properly cited.

\begin{abstract}
Nontuberculous mycobacteria (NTM) are a rare cause of prosthetic joint infections (PJI). However, the prevalence of NTM infections may be increasing with the rise of newer immunosuppressive medications such as biologics. In this case report, we describe a rare complication of immunosuppressive therapies and highlight the complexity of diagnosing and treating PJI due to NTM. The patient is a 79-year-old Caucasian male with a history of severe destructive rheumatoid arthritis on several immunosuppressive agents and right hip osteoarthritis s/p total hip arthroplasty 15 years previously with several complex revisions, presenting with several weeks of worsening right hip and abdominal pain. A right hip CT scan revealed periprosthetic fluid collections. Aspiration of three fluid pockets was AFB smear-positive and grew Mycobacterium avium-intracellulare. The patient was deemed a poor surgical candidate. He underwent a limited I\&D and several months of antimycobacterial therapy but clinically deteriorated and opted for hospice care. PJI caused by NTM are rare and difficult to treat. The increased use of biologics and prosthetic joint replacements over the past several decades may increase the risk of PJI due to NTM. A high index of suspicion for NTM in immunosuppressed patients with PJI is needed.
\end{abstract}

\section{Introduction}

A 79-year-old man with a history of a right total hip arthroplasty (THA) performed 15 years prior to admission was admitted with 3 weeks of right hip pain. His history was notable for severe rheumatoid arthritis (RA), Alzheimer's dementia, coronary artery disease, and several arthroplasty revisions, which were performed 10 and 5 years prior to admission due to dislocations. His RA had been treated with prednisone $15 \mathrm{mg}$ daily, leflunomide $10 \mathrm{mg}$ daily, and infliximab. Six months prior to admission infliximab was replaced with abatacept $500 \mathrm{mg}$ every month.

At presentation, vital signs were normal. BMI was $22.9 \mathrm{~kg} / \mathrm{m}$. He had right lower quadrant tenderness and pain with internal/external rotation of the right lower extremity. His WBC count was within normal limits. The CRP was
$5.2 \mathrm{mg} / \mathrm{L}$ (normal $<3 \mathrm{mg} / \mathrm{L}$ ) and ESR was $28 \mathrm{~mm} / \mathrm{h}$ (nor$\mathrm{mal}<15 \mathrm{~mm} / \mathrm{h}$ ). A CT scan of the abdomen and pelvis revealed complex fluid collections involving the right hip, buttock, and iliac fossa (see Figure 1). A joint aspirate contained 2734 cells $/ \mu \mathrm{L}$ WBC with $89 \%$ neutrophils. Aspirates from his joint and fluid collections were sent for Gram stain and culture, AFB smear and culture, and fungal cultures. Blood cultures were not sent. The AFB smears from each joint and fluid collection resulted positive for acid fast bacilli. The interferon gamma release assay (Quantiferon Gold) was indeterminate. HIV was not checked. His immunosuppression was adjusted, and leflunomide and abatacept were replaced with methotrexate and hydroxychloroquine. On day 14, mycobacterial cultures speciated as Mycobacterium avium-intracellulare (MAI). Rifampin, ethambutol, and clarithromycin were started. 


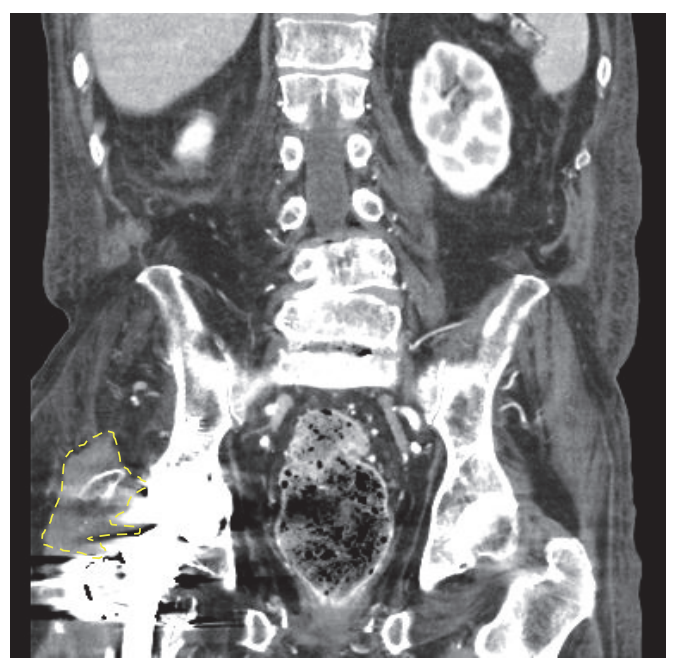

(a)

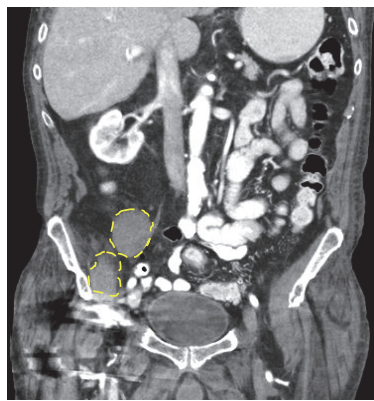

(c)

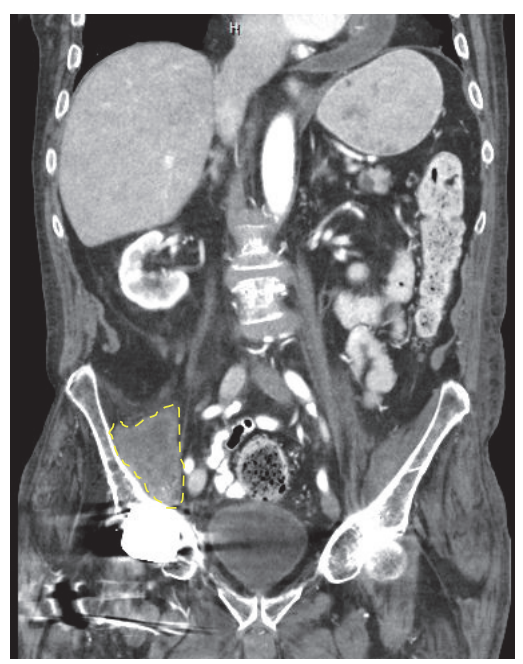

(b)

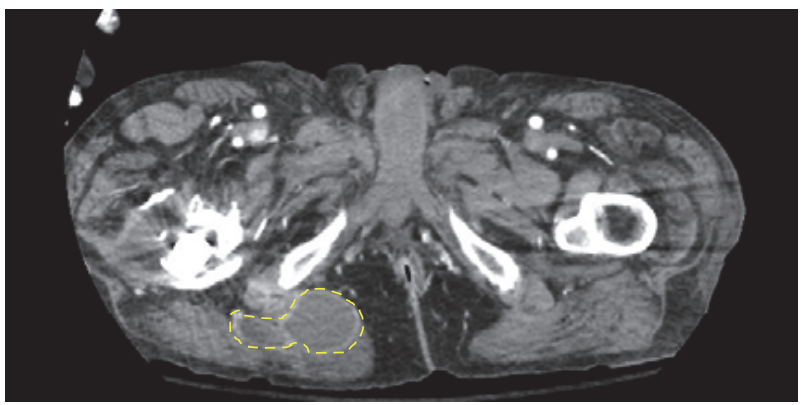

(d)

Figure 1: CT abdomen. (a) Coronal cross section demonstrating a right hip complex fluid collection. (b) Right posterior iliac fossa fluid collection. (c) Right anterior iliac fossa fluid collections. (d) Transverse section demonstrating right buttock fluid collection.

A staged arthroplasty revision was not considered due to his comorbidities. Instead, he underwent extensive incision and drainage (I\&D) of the joint and surrounding tissues, with percutaneous drain placement into the fluid collections. On discharge, he was continued on antimycobacterials for chronic suppression. After 4 months of therapy with ethambutol, clarithromycin, and rifampin, he continued to have positive AFB smears from the surgical site and percutaneous drains. After several months of declining quality of life, he was placed on hospice and died 6 months after initial presentation.

\section{Discussion}

MAI (M. avium and M. intracellulare) are slow growing mycobacteria that typically cause human disease in the form of pulmonary infection among immunocompetent hosts, disseminated infection in the setting of HIV/AIDS, and cervical lymphadenitis [1]. Mycobacteria are a rare cause of prosthetic joint infection (PJI), making up $0-0.6 \%$ of cases, with the majority of these caused by $M$. tuberculosis $[2,3]$. In one retrospective study of PJI caused by rapidly growing mycobacteria (RGM), only eight cases were identified over a 38-year time period, with the majority of cases occurring in immunocompetent hosts [4]. In contrast, PJI due to MAI occurs most often in immunocompromised hosts and is significantly rarer (see Table 1) [2, 5-10]. To the best of our knowledge, this is the 7 th reported case of PJI due to MAI and the first case in which the predisposing factor was immunosuppression due to antirheumatic medications.

In this patient, mycobacteria were likely introduced during a THA revision and persisted for years prior to disease onset in the setting of exogenous immunosuppression [11]. TNF-alpha antagonists, particularly infliximab, predispose patients to mycobacterial infections by blocking macrophage activation and intracellular killing [12]. The patient had risk factors both for PJI in general (RA, exogenous immunosuppressive medications) and for mycobacterial infection specifically, due to the use of multiple disease-modifying antirheumatic drugs (DMARDs). The use of oral corticosteroids and certain DMARDs such as TNF-alpha inhibitors and leflunomide increases the risk of both tuberculous and nontuberculous mycobacterial infections among patients with RA [13]. Abatacept has been associated with tuberculosis; however whether or not the drug is a risk factor for mycobacterial infection is unclear [14].

Making the diagnosis of NTM PJI requires obtaining mycobacterial cultures from clinical specimens in immunosuppressed patients at risk for NTM. Optimal management relies on experience from pulmonary MAI and PJI due RGM. 


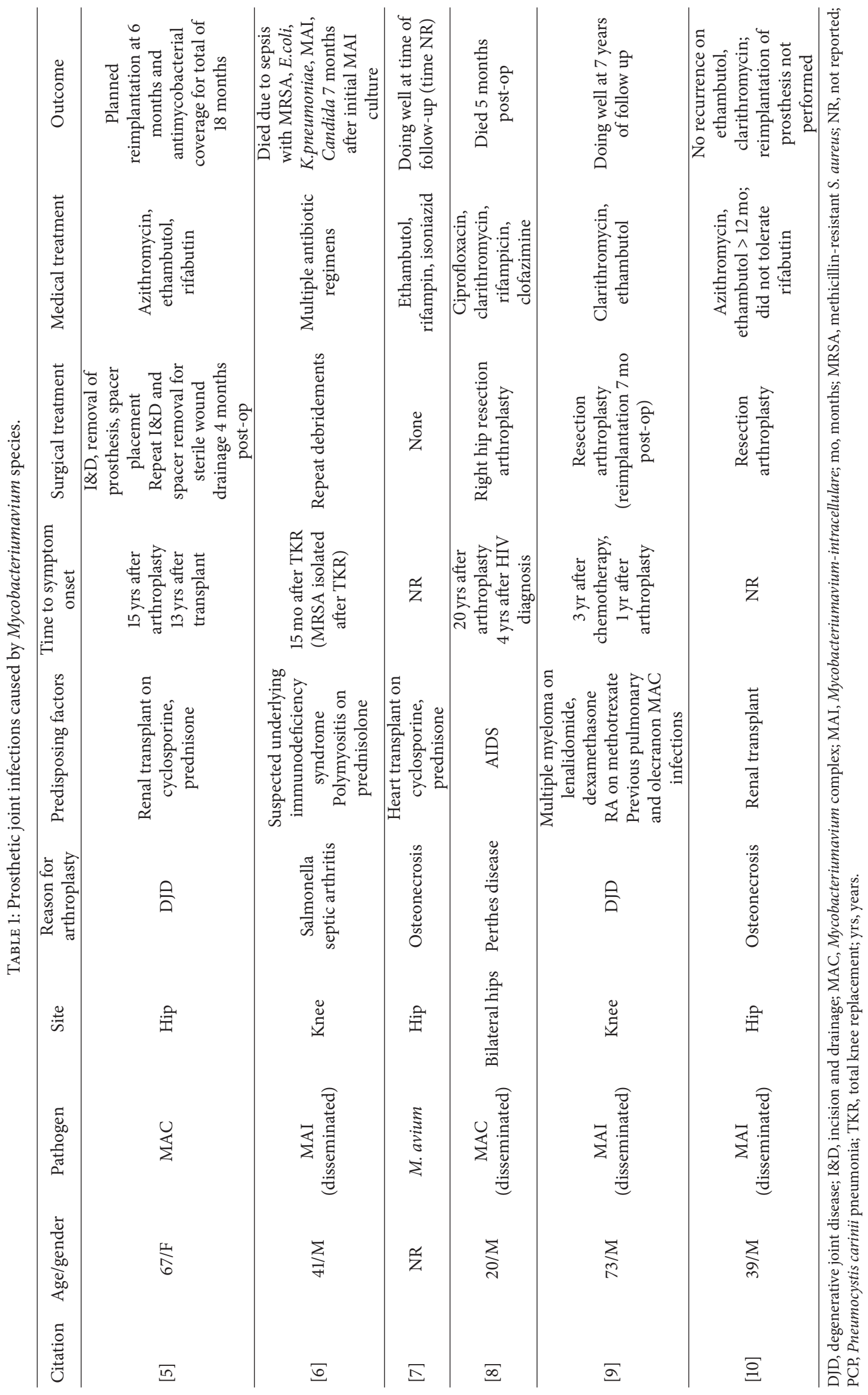


A prolonged course of three drugs (macrolide, ethambutol, and rifampin) is recommended, in addition to surgical debridement or surgical excision [15]. Susceptibilities should be obtained to guide therapy. A case series of patients with PJI due to RGM suggested that resection arthroplasty with appropriate antimycobacterial drugs resulted in the best chance for cure. If debridement with prosthesis retention is performed, prolonged and indefinite antimycobacterial drugs are likely needed to prevent relapse $[2,4,16]$. Similar to PJI caused by rapid-growing NTM species, the prognosis for slow growing NTM PJI is variable (see Table 1 ).

In conclusion, MAI is a rare cause of PJI, occurring almost exclusively in immunocompromised patients. Among patients with PJI taking DMARDs or other immunosuppressive biologic medications, a high index of suspicion is needed for opportunistic infections such as MAI. Ensuring multiple intraoperative specimens is sent for AFB smear/culture is imperative for diagnosis.

\section{Competing Interests}

The authors declare that there is no conflict of interests regarding the publication of this paper.

\section{References}

[1] F. Gordin and R. Horsburgh, "Mycobacterium avium complex," in Principles and Practice of Infectious Diseases, J. E. Bennett, R. Dolin, and M. Blaser, Eds., pp. 2832-2843, Elsevier, Philadelphia, Pa, USA, 8th edition, 2015.

[2] A. J. Tande and R. Patel, "Prosthetic joint infection," Clinical Microbiology Reviews, vol. 27, no. 2, pp. 302-345, 2014.

[3] V. K. Aggarwal, H. Bakhshi, N. U. Ecker, J. Parvizi, T. Gehrke, and D. Kendoff, "Organism profile in periprosthetic joint infection: pathogens differ at two arthroplasty infection referral centers in Europe and in the United States," The journal of knee surgery, vol. 27, no. 5, pp. 399-406, 2014.

[4] A. J. Eid, E. F. Berbari, I. G. Sia, N. L. Wengenack, D. R. Osmon, and R. R. Razonable, "Prosthetic joint infection due to rapidly growing mycobacteria: report of 8 cases and review of the literature," Clinical Infectious Diseases, vol. 45, no. 6, pp. 687-694, 2007.

[5] A. Gupta and H. Clauss, "Prosthetic joint infection with Mycobacterium avium complex in a solid organ transplant recipient," Transplant Infectious Disease, vol. 11, no. 6, pp. 537540, 2009.

[6] C.-H. Hsiao, A. Cheng, Y.-T. Huang, C.-H. Liao, and P.-R. Hsueh, "Clinical and pathological characteristics of mycobacterial tenosynovitis and arthritis," Infection, vol. 41, no. 2, pp. 457-464, 2013.

[7] S. S. Isono, S. T. Woolson, and D. J. Schurman, "Total joint arthroplasty for steroid-induced osteonecrosis in cardiac transplant patients," Clinical Orthopaedics and Related Research, vol. 217, pp. 201-208, 1987.

[8] J. R. McLaughlin, M. Tierney, and W. H. Harris, "Mycobacterium avium intracellulare infection of hip arthroplasties in an AIDS patient," Journal of Bone and Joint Surgery-Series B, vol. 76, no. 3, pp. 498-499, 1994.

[9] E. M. Tan, J. R. Marcelin, E. Mason, and A. Virk, "Mycobacterium avium intracellulare complex causing olecranon bursitis and prosthetic joint infection in an immunocompromised host," Journal of Clinical Tuberculosis and Other Mycobacterial Diseases, vol. 2, pp. 1-4, 2016.

[10] P. Vergidis, T. G. Lesnick, W. K. Kremers, and R. R. Razonable, "Prosthetic joint infection in solid organ transplant recipients: A Retrospective Case-Control Study," Transplant Infectious Disease, vol. 14, no. 4, pp. 380-386, 2012.

[11] J. W. Park, Y. S. Kim, J. O. Yoon et al., "Non-tuberculous mycobacterial infection of the musculoskeletal system: pattern of infection and efficacy of combined surgical/antimicrobial treatment," Bone and Joint Journal, vol. 96, no. 11, pp. 1561-1565, 2014.

[12] K. L. Winthrop, E. Chang, S. Yamashita, M. F. Iademarco, and P. A. LoBue, "Nontuberculous mycobacteria infections and antitumor necrosis factor- $\alpha$ therapy," Emerging Infectious Diseases, vol. 15, no. 10, pp. 1556-1561, 2009.

[13] S. K. Brode, F. B. Jamieson, R. Ng et al., "Increased risk of mycobacterial infections associated with anti-rheumatic medications," Thorax, vol. 70, no. 7, pp. 677-682, 2015.

[14] K. L. Winthrop and M. Iseman, "Bedfellows: mycobacteria and rheumatoid arthritis in the era of biologic therapy," Nature Reviews Rheumatology, vol. 9, no. 9, pp. 524-531, 2013.

[15] D. E. Griffith, T. Aksamit, B. A. Brown-Elliott et al., "An official ATS/IDSA statement: diagnosis, treatment, and prevention of nontuberculous mycobacterial diseases," American Journal of Respiratory and Critical Care Medicine, vol. 175, no. 4, pp. 367416, 2007.

[16] E. F. Egelund, K. P. Fennelly, and C. A. Peloquin, "Medications and monitoring in nontuberculous mycobacteria infections," Clinics in Chest Medicine, vol. 36, no. 1, pp. 55-66, 2015. 


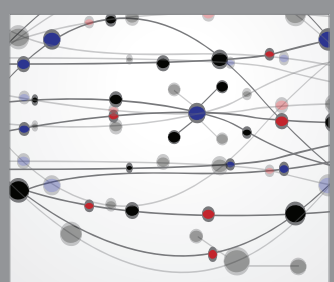

The Scientific World Journal
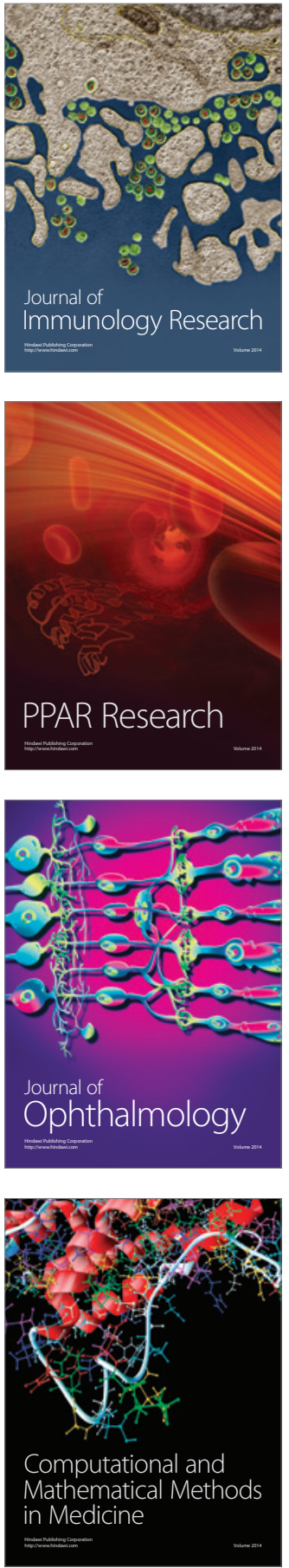

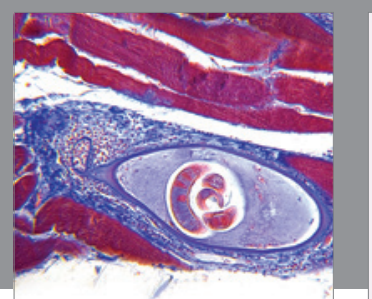

Gastroenterology Research and Practice
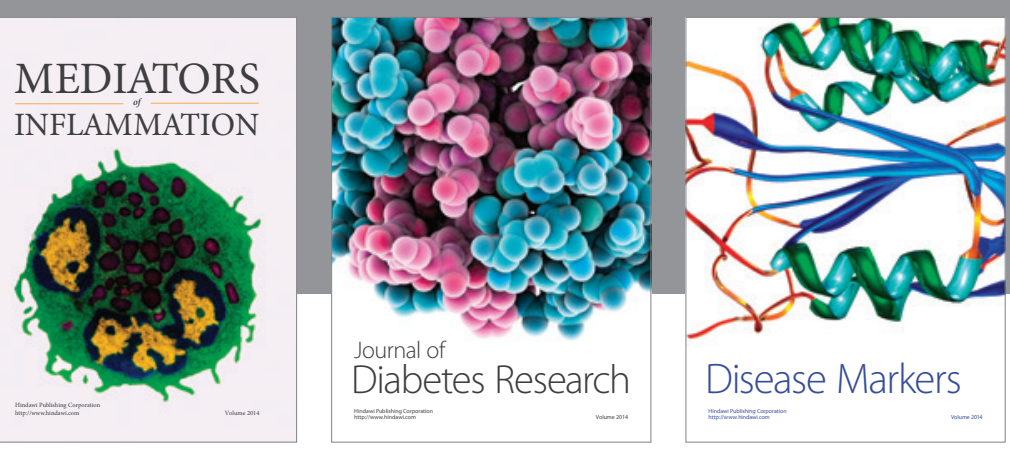

Disease Markers

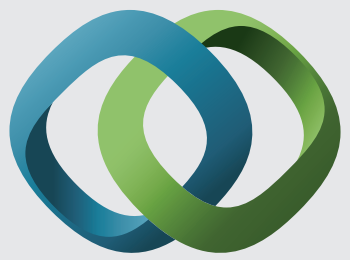

\section{Hindawi}

Submit your manuscripts at

https://www.hindawi.com
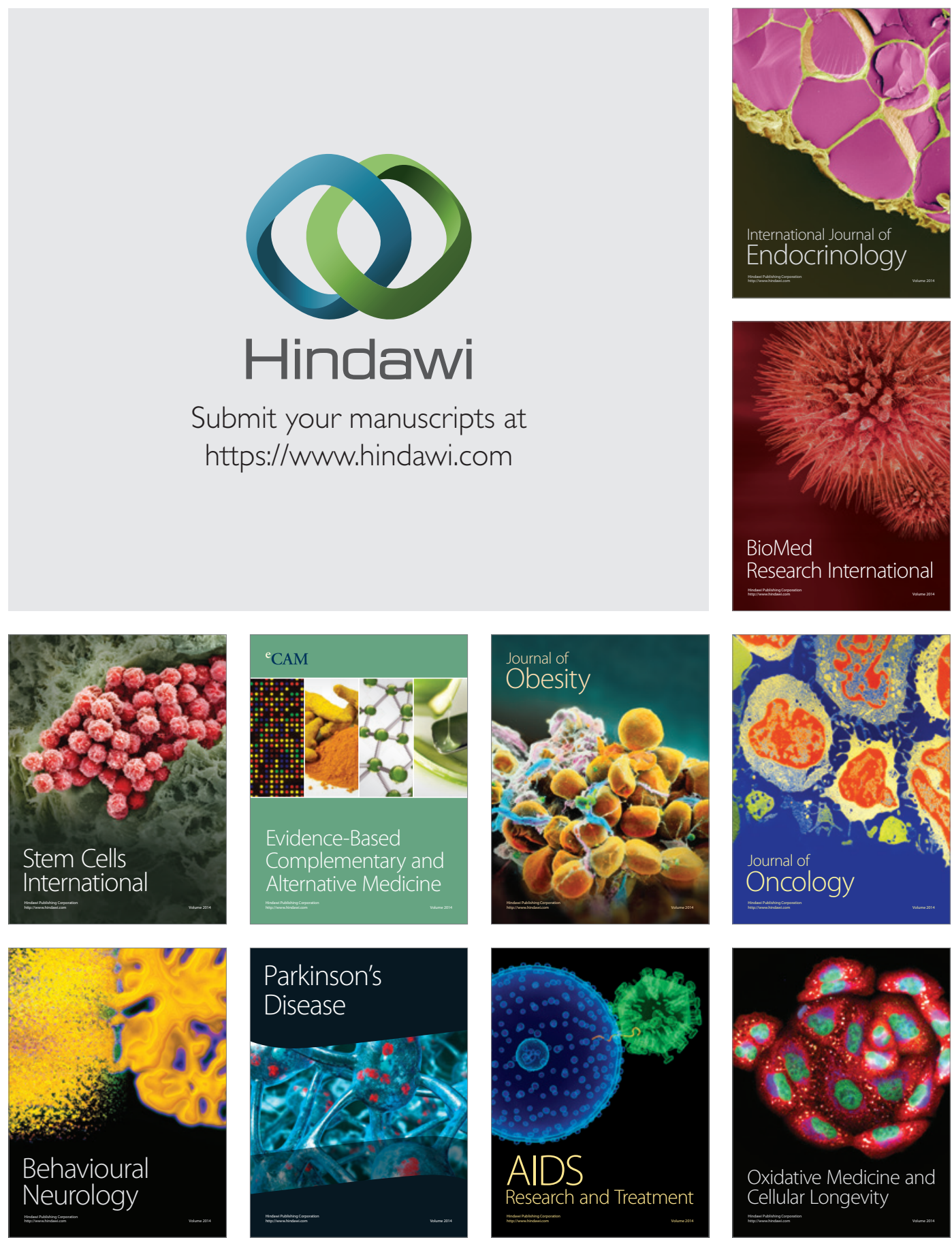